\title{
Gradhiva
}

GRADHIV

Revue d'anthropologie et d'histoire des arts

32 | 2021

Livres sorciers

\section{Le premier livre}

The First Book

\section{Pierre Déléage}

\section{(2) OpenEdition}

Journals

Édition électronique

URL : https://journals.openedition.org/gradhiva/5371

DOI : 10.4000/gradhiva.5371

ISSN : 1760-849X

\section{Éditeur}

Musée du quai Branly Jacques Chirac

\section{Édition imprimée}

Date de publication : 24 mars 2021

Pagination : 48-59

ISBN : 978-2-35744-132-3

ISSN : 0764-8928

\section{Référence électronique}

Pierre Déléage, «Le premier livre », Gradhiva [En ligne], 32 | 2021, mis en ligne le 02 avril 2021, consulté le 25 mai 2021. URL : http://journals.openedition.org/gradhiva/5371 ; DOI : https://doi.org/10.4000/ gradhiva.5371

(c) musée du quai Branly 


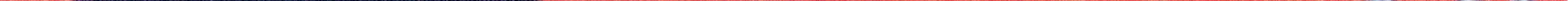


Le premier livre

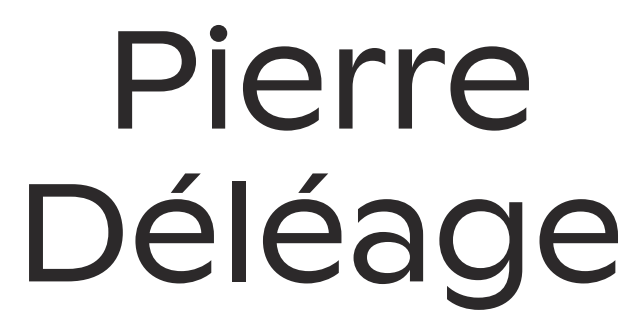


Entre 2000 et 2014, les pratiques chamaniques des Shipibo-Conibo du Pérou se sont transformées. Ce texte insiste sur l'introduction, dans des cérémonies traditionnellement dominées par l'oralité, de livres de magie publiés en espagnol et vendus dans les marchés de la région. Un tel phénomène d'innovation rituelle permet en particulier d'interroger la valeur que certains chamanes shipibo-conibo accordent à l'oral et à l'écrit.

En l'an 2000 j'ai rendu visite à une petite dizaine de communautés d'Amazonie péruvienne, à la recherche d'une tradition orale riche et complexe restée à l'écart de l'écriture et du christianisme. Débarqué dans la région avec des intérêts issus de mes lectures philosophiques plutôt que de ma très maiǵre érudition en ethnologie, je poursuivais quelque chose d'invisible - des paroles stockées dans la mémoire - et non l'univers plus ou moins fantasmatique associé à la forêt tropicale - des arcs et des flèches, des plumes et des peintures corporelles, des pirogues et des huttes, des anacondas et des jaguars. C'est ainsi que je me retrouvai presque par hasard dans le village de Nuevo San Martín, sur le fleuve Inuya, chez les Amahuaca qui me reçurent avec une bienveillance dont je mesure maintenant combien elle était charitable vis-à-vis d'un jeune étranger perdu, incapable et inutile. La vie y était aǵréable, peut-être un peu ennuyeuse, mais je n'avançais pas dans ma recherche: le répertoire de Pedro, le vieil homme amahuaca dont tous disaient qu'il était le plus savant de la communauté, était limité à quelques brefs chants propitiatoires qui n'excitèrent pas démesurément ma curiosité.

L'étape suivante fut le village de Huayhuashi où je trouvai ce que j'étais venu chercher. Raimundo, le chamane de cette communauté yaminahua, paraissait disposer d'un riche savoir et quand je me rendis compte qu'ils étaient plusieurs à se réunir, la nuit tombée, pour chanter les visions hallucinées déclenchées par l'absorption d'un hallucinogène, je sus que ma quête approchait de sa fin. Je ne fus définitivement convaincu que lorsque j'appris que les chants rituels étaient formulés en une lanǵue secrète que ne pouvaient comprendre que les chamanes et les anacondas. Le fils de Raimundo était par contre très moyennement enthousiasmé par ma présence et un jour de beuverie je reconnus qu'à défaut d'une autre tradition - celle des Yaminahua semblait idéale pour mon projet - il me faudrait au moins trouver une autre communauté. Je la trouverais plus tard et bien plus loin, sur le cours supérieur du fleuve Purús, chez les Sharanahua.

Mais auparavant je décidai de rendre visite à une collègue, Frédérique Rama Leclerc, qui séjournait alors dans la communauté de San Francisco, sur la rive de la laǵune de Yarinacocha, à quelques kilomètres de la ville de Pucallpa. Elle menait une enquête sur les connaissances botaniques des Shipibo-Conibo, l'un des peuples les plus importants d'Amazonie péruvienne, qui compte à ce jour quelque trente-cinq mille personnes et occupe une position centrale le long du fleuve Ucayali. Elle me présenta à l'un des chamanes dont elle étudiait le savoir, Edinson Ramos, qui eut la gentillesse de m'inviter à une de ses cérémonies thérapeutiques (une fois l'accord des patients obtenu). La communauté shipibo de San Francisco était particulièrement intéressante durant cette période puisqu'on pouvait y observer la naissance d'une nouvelle forme de chamanisme, orientée vers une clientèle plus large, incluant les migrants habitant à Pucallpa, qui recommençaient à arriver de la côte après un long ralentissement dû à la dangerosité de l'unique route d'accès, et les touristes occidentaux de passage. Le phénomène prit des dimensions spectaculaires quelques années plus tard avec la médiatisation hollywoodienne de Guillermo Arévalo, futur acteur du Blueberry (2004) de Jan Kounen et néanmoins chamane shipibo qui, lorsque je me rendis chez lui, avait déjà quitté sa communauté, ne vivant que des ressources - non négligeables - issues de son commerce chamanique avec les étrangers.

Edinson Ramos Vasquez appartenait à une autre génération. Jeune maître d'école ayant brièvement séjourné dans les Andes, à Chavín de Huántar, il prit rapidement conscience de l'intérêt que les étrangers accordaient de plus en plus au chamanisme shipibo - ne se contentant plus des productions artisanales qui restent un pôle d'attraction majeur de San Francisco, à tel point qu'on se croirait, à en arpenter les rues, dans un village d'artistes: les façades des maisons y sont souvent couvertes des motifs géométriques traditionnels (nommés quene), les fours à céramique parfois sculptés en forme de tête humaine, la plupart des femmes occupées à broder, à teindre, à coudre, à peindre, à mouler ou à enfiler des perles, certains hommes ciselant des morceaux de bois et alimentant les fours. Plutôt que de rejeter les connaissances qu'il avait acquises à l'école de formation des maîtres, Edinson choisit de combiner les compétences de ses deux univers culturels. Il avait ainsi fondé avec quelques amis une association de médecine traditionnelle, dans l'idée d'obtenir des financements de l'étranger: l'Asociación de Médicos Indígenas de Aplicación e Investigación de Medicina Tradicional. 


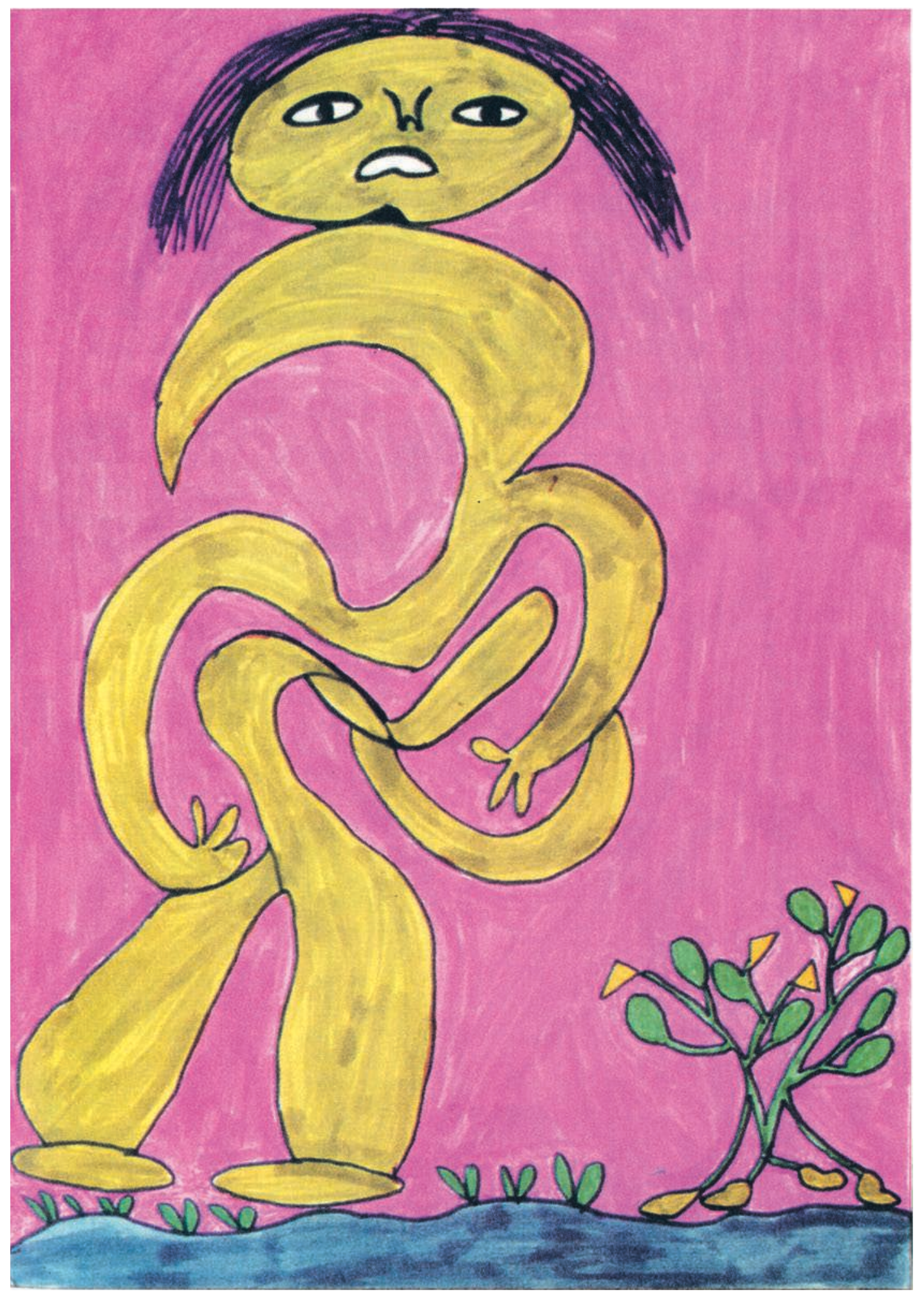

Lastenia Canayo, Maître de l'arachide, in Los dueños del mundo Shipibo-Konibo 2004, Universidad Nacional Mayor de San Marcos, p. 41.

Cet ouvrage étonnant n'aurait pas vu le jour sans le soutien institutionnel du Seminario de Historia Rural Andina qui, à partir des années 1990 , a activement contribué à l'émergence

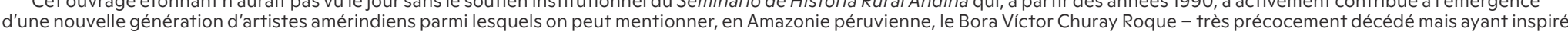
de nombreux jeunes, dont Brus Rubio Churay -, I'Asháninca Enrique Casanto Shingari ou les Shipibo Elena Valera, Roldán Pinedo Lopez et Harry Pinedo. 

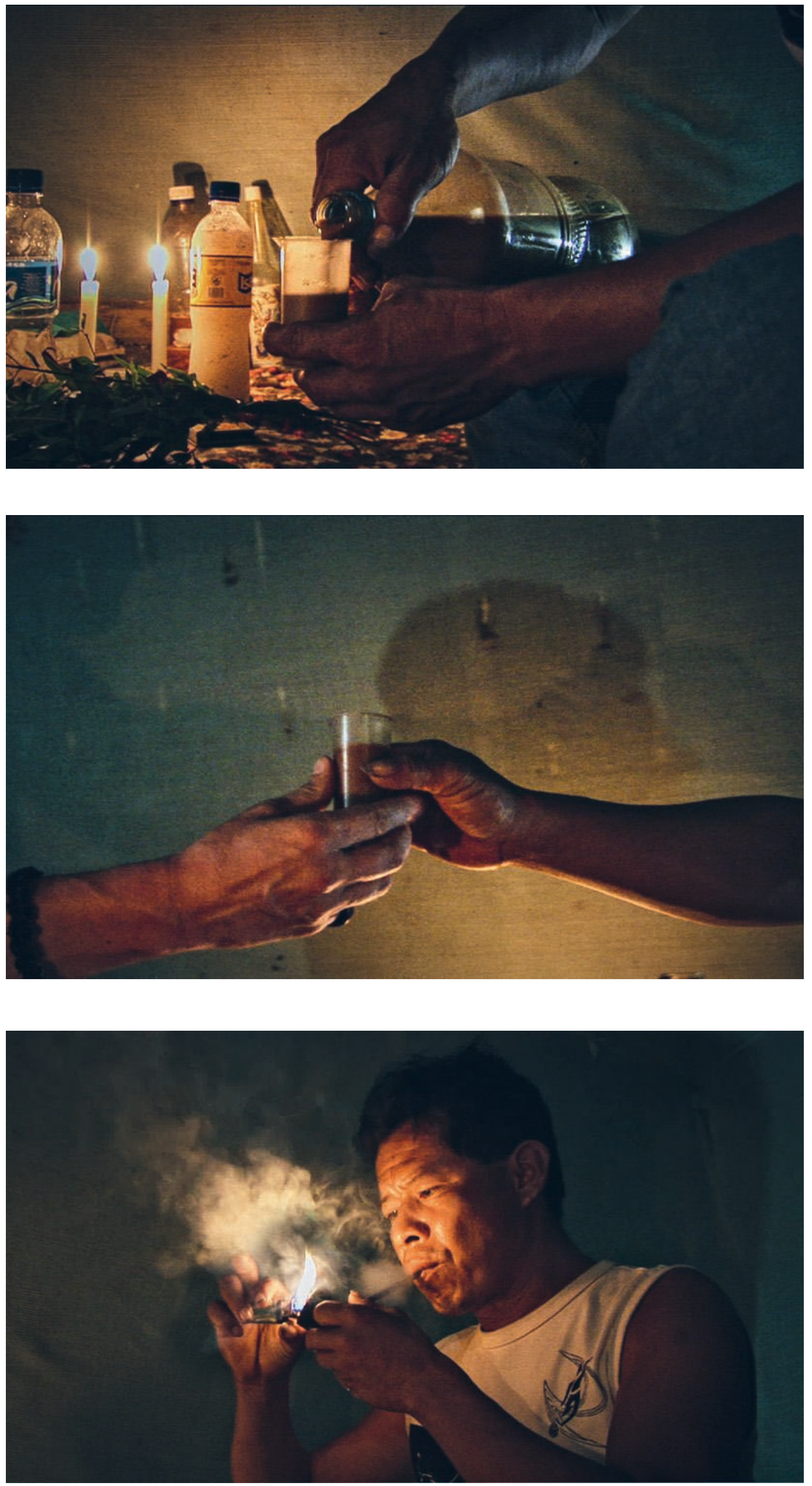

Photogrammes du film Spur der Geister («Sentier des Esprits»), 2011, montrant Edinson Ramos lors d'une cérémonie d'ayahuasca. Réalisation Martin Weinhart, images Pascal Hoffmann. Avec l'aimable autorisation de Martin Weinhart et Pascal Hoffmann. 
La cérémonie chamanique à laquelle j'assistai était très différente de celle des Yaminahua. Je me souviens en particulier d'une surprenante atmosphère de recueillement et d'une attention très scolaire à certains détails, tels que le silence (la cérémonie ne commença que vers 10 heures, lorsque s'éteignit la musique diffusée par les haut-parleurs installés dans tout le village), l'esthétique traditionaliste (l'officiant était vêtu d'une cushma peinte de motifs quene) ou l'ordre des choses (une série d'objets maǵiques étaient précisément rangés dans une disposition immuable). En dehors de cela, il était classiquement question de consommer un hallucinogène - le même que les Yaminahua, l'ayahuasca -, de chanter et de fumiger le corps des patients, procédure à peu près standardisée dans la région.

Ce qui retint toutefois mon attention, ce fut l'ouverture de la cérémonie. Edinson Ramos fouilla son sac, en sortit avec délicatesse un livre de petit format et commença à lire, en espaǵnol, une des prières qu'il contenait. Le livre, fatigué, était plutôt mal imprimé et sa couverture aux couleurs criardes affichait un dessin kitsch que j'ai oublié. J'écrivis à l'époque que «les prières du livre, appels à Dieu ou au Christ pensés comme les forces les plus puissantes de la nature, étaient considérées comme les plus aptes à guérir». Selon Rama Leclerc, cette hiérarchie entre les entités surnaturelles - les esprits des animaux et des végétaux apparaissant comme des forces inférieures créées par Dieu et les saints - reflétait le conflit qui opposait les anciens chamanes aux jeunes, influencés par l'école et l'Éǵlise'.

Mais en marge de ce phénomène générationnel, je restai songeur devant cet usage d'un livre dans le rituel, usage qui contrastait tellement avec ce que j'étais venu chercher - une tradition «purement» orale. Quelques semaines plus tard, j'achetai deux livres maǵiques semblables à ceux utilisés par Edinson dans une papeterie de Tingo María, un Pequeño libro de san Cipriano et un Escudo sagrado contra los demonios, las brujas y los maleficios. Je remettais à plus tard l'étude de cette nouvelle forme de transmission, duelle, où l'oral avait ménagé une vacuole à l'écrit. J'imaginais un avenir proche où les chamanes shipibo délaisseraient peu à peu la récitation par cour des chants traditionnels au profit d'une consultation ponctuelle et d'une lecture des prières espaǵnoles imprimées dans les livres de maǵie.

En mars 2014, je me rendis de nouveau chez Edinson Ramos. La ville de Pucallpa avait beaucoup changé en dix ans. Si les bâtiments abandonnés du Cine Rex et du Cine Ucayali trônaient toujours au centre de la ville, c'étaient les derniers témoins du marasme qu'elle connut pendant un temps. Tout avait été rénové, les routes, la place des armes, le port, les édifices publics, l'aéroport - les murs étaient repeints de frais, on ajoutait des étages aux maisons, on parlait même d'éradiquer les anciens marchés infestés de chiens errants. Des galeries commerciales, des cinémas multiplex, des supermarchés, une fête foraine et même une rue piétonne avaient fait leur apparition. On net- toyait les feuilles des plantes décoratives de la place de la mairie au savon. La population avait triplé, le volume de la circulation aussi. L'heure était à la croissance insoutenable, à l'exploitation décomplexée et dévastatrice de la forêt, si constamment encouragée par les gouvernements d'Alan García puis d'Ollanta Humala, au grand dam des écologistes et d'une bonne partie des Amérindiens du pays.

La communauté de San Francisco, encore située à l'écart de la ville, n'avait quant à elle pas changé de manière visible. Lorsque je m'y rendis, la route n'était pas praticable, à cause de la crue du fleuve, et il me fallut emprunter le service de bateaux. Ma voisine, artisane de retour du quartier Cantagallo à Lima où vivaient quelque deux mille Shipibo -, me décrivit pendant le trajet les différents changements intervenus dans le village, m'apprit que la diaspora shipibo s'étendait maintenant à peu près à tous les sites touristiques du Pérou et, malǵré les pétarades assourdissantes du moteur, récita quelques chants en suivant du bout du doigt les motifs quene brodés sur un tissu. Elle me dit que ces motifs étaient l'alphabet des Shipibo, que les textiles étaient des livres et qu'il était possible de lire les chants qu'ils contenaient, arguments de vente que, dubitatif, j'avais déjà entendus quelques fois ${ }^{2}$.

En quatorze ans Edinson Ramos avait changé de statut. Il faisait à présent cavalier seul, ayant abandonné l'association qu'il avait fondée. Il s'était quelque peu retiré: la dernière fois que je l'avais rencontré, il vivait dans une maison au centre de la communauté, à proximité du local de l'association. Il était maintenant installé sur les quatre hectares de terrain de ce qui avait été son essart, à l'extrémité nord du village. Il entretenait depuis six ans un vaste jardin botanique riche, selon ses estimations, de deux cent vinǵt variétés de plantes et il avait fait construire une agréable maison, un logement pour les patients et un large édifice cérémoniel entièrement protégé par une moustiquaire géante. L'herbe était coupée, le terre-plein balayé, les affaires rangées - certains traits de personnalité peuvent persister indéfiniment. Plus tard, je trouvai sur YouTube un documentaire allemand où on voit Edinson officier dans ce nouvel environnement ${ }^{3}$.

Quand je lui parlai de mon voyage en bateau et des chants de ma voisine, il observa un silence puis me confia que sans vouloir critiquer les habitants du village, ils étaient tout de même prêts à faire n'importe quoi pour accroître la valeur marchande de leur production artisanale. Je lui expliquai ensuite que je me souvenais nettement de la manière dont il lisait autrefois, à l'ouverture du rituel, des livres maǵiques. Il me répondit qu'il avait cessé de les utiliser: il connaissait maintenant par cœur les prières espagnoles qu'il jugeait importantes et il était passé à une autre phase de son apprentissage.

Avec un ton professoral et une attitude corporelle à la fois douce et autoritaire, il me tint en espaǵnol un discours dont je pouvais deviner qu'il l'avait répété mille fois. Les livres magiques appartenaient au savoir spirituel, un savoir clos, limité, par lequel il était important d'être passé mais qui n'était en rien suffisant. Les plantes, elles, appartenaient au savoir terrestre
1. Rama Leclerc 2004; voir aussi désormais: Caruso 2005; Colpron 2012; Déléage 2016; Slaghenauffi 2019.

2. Voir Déléage 2017: 102-106.

3. Film Spur der Geister («Sentier des Esprits»), 2011, réalisé par Martin Weinhart, images Pascal Hoffmann, disponible en ligne: https:// disponible en ligne: $\mathrm{h}$ th watch?v $=\mathrm{c} 4 \mathrm{H} 84 \mathrm{HOq}-8 \mathrm{~s}$ 


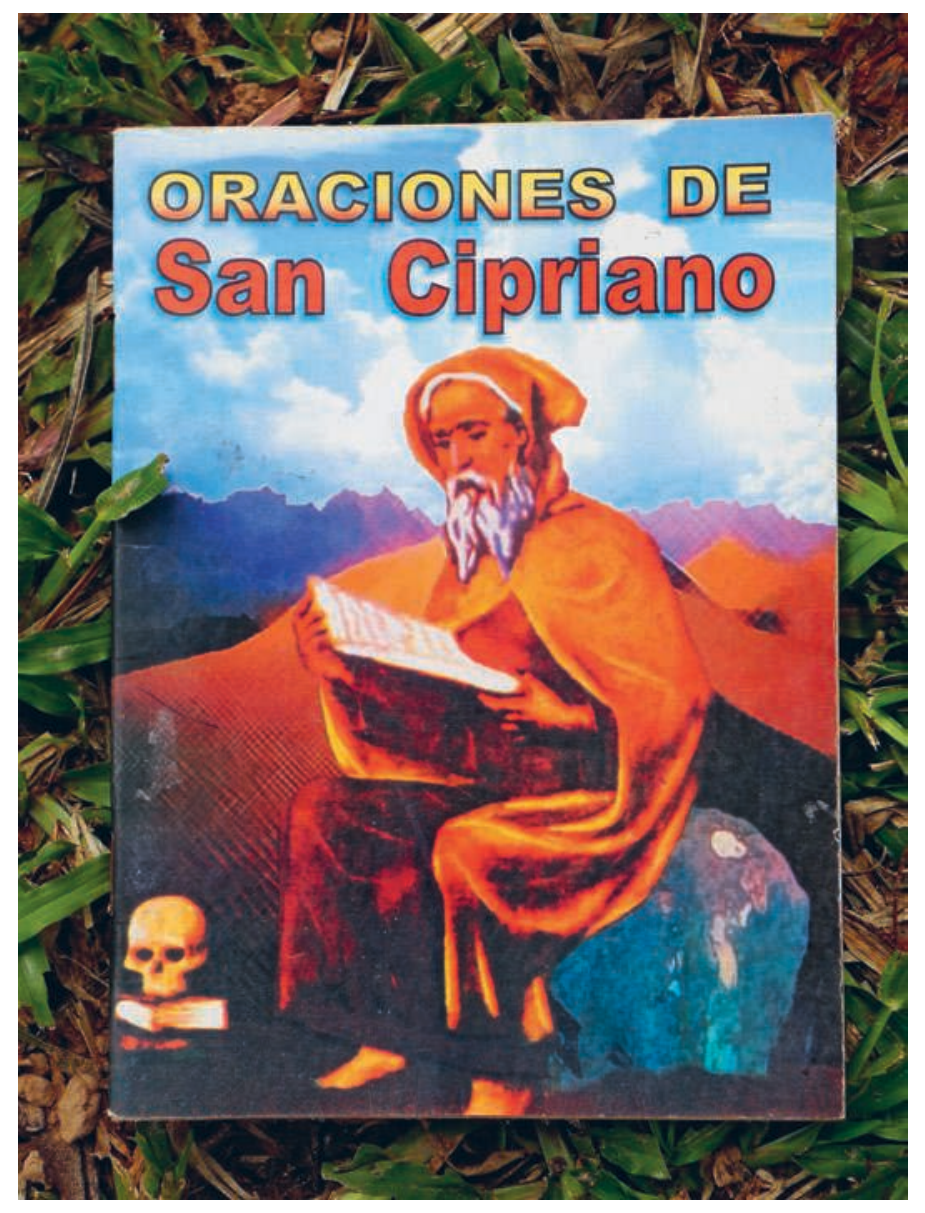

Exemplaire du Livre de Saint Cyprien appartenant à Edinson Ramos. Photographie de Pierre Déléage, 2014.

et ce savoir était infini. Par «savoir des plantes», il ne fallait pas seulement penser à l'art de reconnaître et d'utiliser une vaste taxonomie végétale, il fallait aussi inclure un large répertoire de chants originellement transmis par les esprits des plantes et des animaux, chants qu'Edinson avait appris de son père et de sa tante. Avec les années le conflit générationnel s'était apaisé, le savoir livresque en sortait légèrement dévalué tandis que le savoir, disons, oral, était revenu sur le devant de la scène. Mes prévisions étaient déjouées. Il est possible que la féroce concurrence qui oppose dorénavant les chamanes de San Francisco entre eux soit une raison de ce relatif abandon: pour la nouvelle génération, les livres sont aisément accessibles, tandis que le savoir oral, moins tanǵible, est plus susceptible de conférer une réelle distinction charismatique. De plus, je pense que la clientèle étrangère ne voyait pas d'un très bon œil ces ouvrages aux couvertures souvent un peu ridicules qui ne correspondaient pas à la sagesse ancestrale qu'ils étaient venus chercher si loin de chez eux.

Edinson Ramos me montra ensuite sa bibliothèque privée. Il me prévint d'abord qu'il ne possédait pas les livres rouge, noir et blanc qui se rapportaient chacun à une maǵie maléfique, sorcellaire, qu'il s'interdisait d'employer. Aux côtés d'un livre de tarot et d'une compilation de «recettes pour se faire aimer», je découvris un exemplaire des Milagros y oraciones de la Cruz de Caravaca et surtout deux éditions du fameux grimoire de saint Cyprien, le Gran libro de san Cipriano (El tesoro del hechizero) et les Oraciones de san Cipriano. Edinson me dit qu'il lui en manquait à présent un ou deux, dont le très important Escudo sagrado. Plus tard il ajouta, au détour d'une autre conversation, qu'il existait dix livres importants : Oraciones de san Cipriano, La Cruz de Caravaca, Escudo sagrado, El Cielo abierto, El cantar de los cantares et El diamante, celui qui contient les secrets les plus fondamentaux. Des quatre autres livres il ne souhaita pas parler, s'étant déjà peut-être un peu trop avancé. Quand je lui demandai où il avait acheté ses exemplaires, il fit quelques réponses évasives puis finit par me dire qu'ils venaient de la lointaine Lima.

Tous ces livres imprimés sont des recueils de formules, de prières et de chants parfois agrémentés de symboles talismaniques. J'avoue ne pas en avoir réalisé d'étude approfondie. Ils me semblent interchangeables et je pense qu'ils sont le plus souvent évalués à l'aune de leur couverture: si l'on y voit dessiné quelque chose de vaguement monstrueux, on aura tendance à l'associer à la sorcellerie satanique, tandis qu'un simple symbole catholique évoquera la guérison. Je ne doute pas qu'il doit exister certains usages plus érudits mais il m'a paru que c'était ainsi, de manière autodidacte, qu'Edinson avait choisi ses livres et ses prières. Le Livre de saint Cyprien contenait selon lui les prières dont l'usage était le plus courant lors des cérémonies thérapeutiques.

Il vaut peut-être la peine de rappeler que saint Cyprien d'Antioche est un légendaire martyr chrétien du $\mathrm{III}^{\mathrm{e}}$ siècle. Considéré dès le Moyen Âge comme un 


\section{Le premier livre}

Pierre Déléage

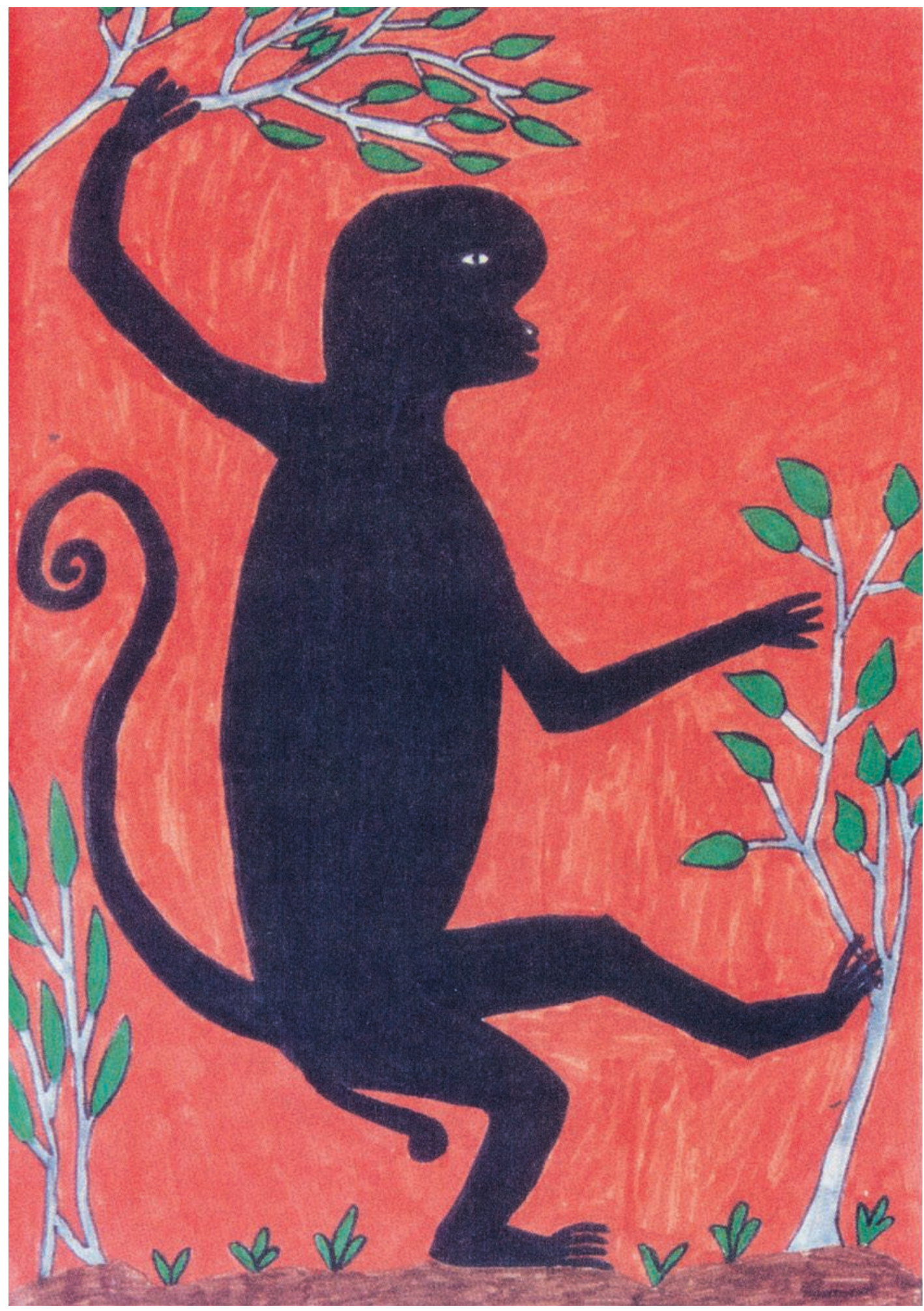

Lastenia Canayo, Le Diable du Maquisapa, in Los dueños del mundo Shipibo-Konibo 2004, Universidad Nacional Mayor de San Marcos, p. 53. 

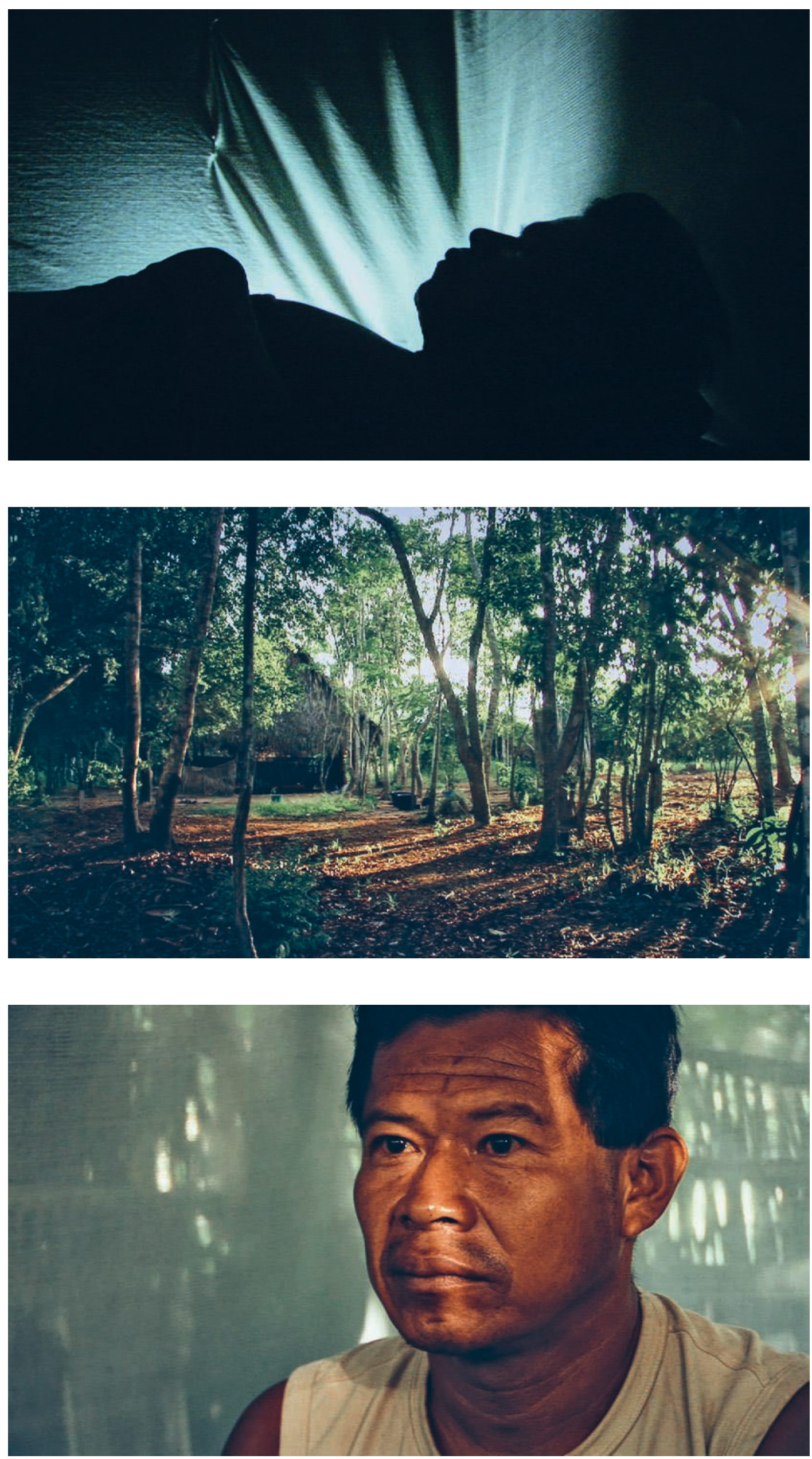

Photogrammes du film Spur der Geister («Sentier des Esprits»), 2011, montrant Edinson Ramos lors d'une cérémonie d'ayahuasca. Réalisation Martin Weinhart, images Pascal Hoffmann. Avec l'aimable autorisation de Martin Weinhart et Pascal Hoffmann. 


\section{Le premier livre}

Pierre Déléage

puissant nécromancien, on lui attribua très tôt de nombreux sortilèges. On trouve le nom de saint Cyprien sur les premiers livres de magie à avoir été imprimés - dès 1498 -, et le succès de leur diffusion eut pour conséquence un bannissement par l'Église catholique quelques décennies plus tard. Les éditions originales des livres qu'utilisait Edinson datent toutefois très probablement du XIX ${ }^{\mathrm{e}}$ siècle, lorsque parut sous le titre de Libro de san Cipriano un grimoire en espaǵnol compilant de nombreux textes (dont le Grimoire du pape Honorius). Très populaire en Galicie, il suivit les migrants en Amérique et on le retrouve, au milieu du $\mathrm{Xx}^{\mathrm{e}}$ siècle, dans la santeria cubaine ou dans l'umbanda et le candomblé brésiliens. Les éditions se firent dès lors locales et se répandirent dans l'Amazonie, d'abord à travers le chamanisme des métis, puis dans les Andes. Dans les années 1970 le Libro de san Cipriano était utilisé par les chamanes du fleuve Putumayo à la frontière de la Colombie, de l'Équateur et du Pérou. Il existe également une trilogie de livres intitulés La magia neǵra, La magía blanca et La maǵia roja auxquels, je pense, Edinson faisait référence. Ils sont attestés au Pérou au moins à partir des années 1960 et ils proviendraient du Mexique ${ }^{4}$.

On trouve, dans diverses éditions, tous les livres évoqués ou utilisés par Edinson, ainsi que d'autres comme La magia verde ou le Libro del mago negro, dans une des allées du Mercado Dos en plein cœur de Pucallpa - l'un des marchés qu'il est d'ailleurs question de fermer. Une dizaine de petits étals y proposent des plantes, des décoctions, des crânes, squelettes et autres cuirs d'animaux, toute une variété de substances plus ou moins licites, en somme un paradis aux odeurs saumâtres où l'on croise des vieillards bossus au regard louche et de jeunes hommes frissonnant malgré la chaleur étouffante. Il suffit de demander où l'on peut acheter des livres pour se voir indiquer l'éventaire du fond de l'allée. Là, le vendeur monte sur un tabouret et attrape sur une des étagères les plus haut placées un sac en plastique dissimulé derrière une étole aux symboles cabalistiques, puis d'un air grave, le regard insistant, il en extrait un à un les ouvrages en lisant à chaque fois le titre d'une voix caverneuse. Mi-interloqué, mi-amusé par cette stratégie commerciale plutôt grossière, je finissais par demander, après les avoir feuilletés, le prix de ces grimoires - il était prohibitif, le triple de ce que l'on paie pour des livres de format similaire mais de contenu plus ordinaire.

La diffusion du Libro de san Cipriano et des livres de magie en général dans les Amériques indiennes fut corrélée au degré de bilinguisme et d'alphabétisation des populations qui se les approprièrent. Edinson Ramos fit ainsi partie de la première génération qui introduisit les livres de magiie dans le chamanisme shipibo, à un moment clef où se combinèrent plusieurs processus : l'accès de plus en plus généralisé à l'école et donc à l'écriture, un contact peut-être un peu plus fraternel avec les guérisseurs métis, et une revalorisation du chamanisme shipibo qui, d'un archaïsme issu du passé ou, pire, d'une pratique démoniaque, devint une médecine alternative susceptible d'intéresser, en plus d'une clientèle locale, toute une gamme d'étrangers.
À son tour Edinson Ramos avait su acclimater le savoir livresque aux traditions essentiellement orales du chamanisme de son peuple, phénomène en voie de généralisation dans toute l'Amazonie. Il en était toutefois revenu, préférant au prestige ésotérique des grimoires imprimés l'autorité invisible des esprits de la forêt.
4. Davies 2009; Henningsen 1994; Sur La magia negra, La magia blanca, La magia roja et La magia verde au Pérou, voir: Dobkin 1969.

Sur La Cruz de Caravaca dans le chamanisme métis, voir: Luna 1986. Sur le Libro de san Cipriano et La Santa Cruz de Caravaca dans le chamanisme du Putumayo des années1970 Laboratoire d'anthropologie sociale voir: Taussig 1987. 


\section{Bibliographie}

\section{Caruso, Giuseppe}

2005 Onaya Shipbo-Conibo: sistema médico tradicional y desafios de la modernidad. Quito, Abya-Yala.

\section{Colpron, Anne-Marie}

2012 «Fluctuations et

persistances chamaniques parmi les Shipibo-Conibo de l'Amazonie occidentale», in Marie-Pierre Bousquet

et Robert R. Crépeau (dir.), et Robert R. Crépeau (dir.) Dynamiques religieuses des autochtones des Amériques Paris, Karthala : 387-416.

\section{Davies, Owen}

2009 Grimoires: A History of Magic Books. Oxford, Oxford University Press.

\section{Déléage, Pierre}

2016 «Retour à Pucallpa »,

Hypalampuses Hemeras,

Gruppen 11: 34-42.

2017 Lettres mortes.

Paris, Fayard.

\section{Dobkin, Marlene}

1969 «Fortune's Malice: Divination, Psychotherapy, and Folk Medicine in Peru», Journal of American Folklore 82 (324): 132-141.

\section{Henningsen, Gustav}

1994 «La evangelización neǵra: difusión de la maǵia europea por la América colonial », Revista de la Inquisición 3: 9-27.

\section{Luna, Luis Eduardo}

1986 Vegetalismo: Shamanism Among the Mestizo Population of the Peruvian Amazon. Stockholm, Almquist \& Wiksell International.

\section{Rama Leclerc, Frédérique}

2004 «Shipibo : rapport anthropologique », Tsemim: Transmission et transformation des savoirs sur l'environnement en milieu indigène et métis.

\section{Slaghenauffi, Doriane}

2019 «Korikiati : comment les chamanes shipibo d'Amazonie péruvienne pensent le tourisme, le prosélytisme protestant et l'esprit du capitalisme. » Thèse de doctorat, université Aix-Marseille,

sous la direction de

Frédéric Saumade.

\section{Taussig, Michael}

1987 Shamanism, Colonialism, and the Wild Man: A Study in Terror and Healing. Chicago, University of Chicago Press. 


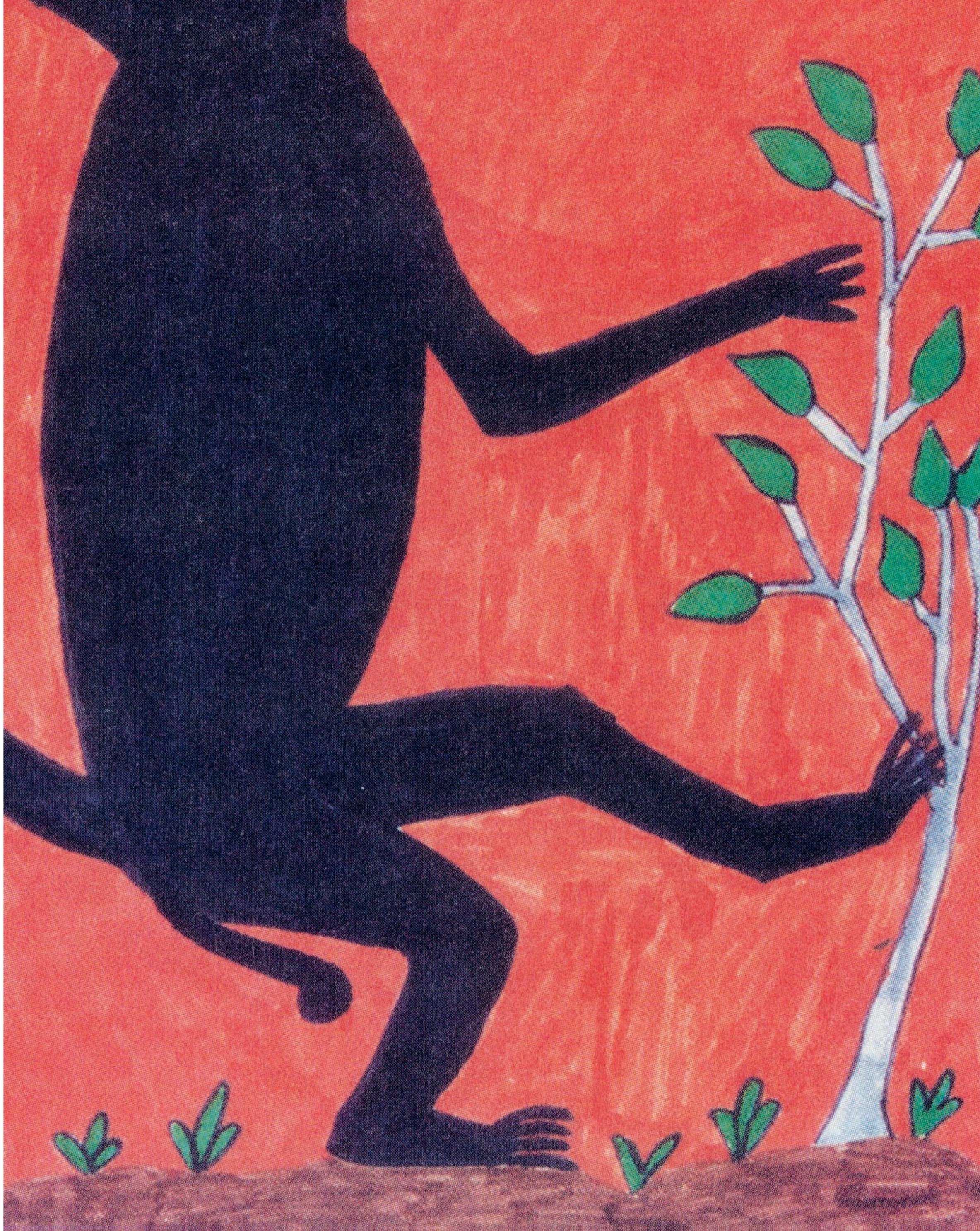

